\title{
Acute and subacute toxicity profiles of thymoquinone-loaded nanostructured lipid carrier in BALB/c mice
}

This article was published in the following Dove Press journal:

International Journal of Nanomedicine

9 November 2016

Number of times this article has been viewed

\author{
Yong Sze Ong' \\ Latifah Saiful Yazan ${ }^{1,2}$ \\ Wei Keat $\mathrm{Ng}^{\prime}$ \\ Mustapha M Noordin ${ }^{3}$ \\ Sarah Sapuan' \\ Jhi Biau Foo' \\ Yin Sim Tor ${ }^{1}$
}

'Laboratory of Molecular Biomedicine, Institute of Bioscience, ${ }^{2}$ Department of Biomedical Science, Faculty of Medicine and Health Sciences, ${ }^{3}$ Department of Pathology and Veterinary Microbiology, Faculty of Veterinary Medicine, Universiti Putra Malaysia, Selangor, Malaysia
Correspondence: Latifah Saiful Yazan Department of Biomedical Science, Faculty of Medicine and Health Sciences, Universiti Putra Malaysia, UPM Serdang 43400, Selangor, Malaysia

Tel +60389472308

Fax +60389436178

Email latifahsy@upm.edu.my
Background: Thymoquinone (TQ), the predominant active lipophilic component in Nigella sativa seed oil, has a variety of pharmacological properties such as anticancer activities. However, translation of TQ to clinical phase is still not possible due to its hydrophobic properties. This problem can be solved by encapsulating it in nanoformulations to enhance its pharmacological properties. In our previous study, TQ has been successfully encapsulated in a nanostructured lipid carrier (hereinafter referred to as TQNLC) with excellent physiochemical properties such as high encapsulation efficiency, high drug-loading capacity, particle diameter less than $100 \mathrm{~nm}$, and stability up to 2 years. In vitro studies also proved that TQNLC exhibited antiproliferative activity toward breast and cervical cancer cell lines. However, no toxicity profile related to this formulation has been reported. In this study, we determine and compare the in vivo toxicity of both TQNLC and TQ.

Materials and methods: The in vivo toxicity (acute and subacute toxicity) study was carried out by oral administration of TQNLC and TQ to BALB/c mice. Animal survival, body weight, organ weight-to-body weight ratio, hematological profile, biochemistry profile, and histopathological changes were analyzed.

Results: In acute toxicity, TQ that is loaded in nanostructured lipid carrier (NLC) was found to be less toxic than pure TQ. It can be concluded that encapsulation of TQ in lipid carrier minimizes the toxicity of the compound. In the subacute toxicity study, oral administration of $100 \mathrm{mg} / \mathrm{kg}$ of TQNLC and TQ did not cause mortality to either male or female but resulted in toxicity to the liver. It is postulated that long-term consumption of TQNLC and TQ may cause toxicity to the liver but not to the extent of altering the functions of the organ. For both treatments, the no observed adverse effect level (NOAEL) was found to be $10 \mathrm{mg} / \mathrm{kg} / \mathrm{d}$ for mice in both sexes.

Conclusion: For long-term oral consumption, TQ and TQNLC at a dose of $10 \mathrm{mg} / \mathrm{kg}$ is safe in mice and does not exert any toxic effect. The results provide safety information of TQNLC, which would further help researchers in clinical use.

Keywords: thymoquinone, nanostructured lipid carrier, toxicity

\section{Introduction}

Nigella sativa, commonly known as black cumin, is an annual herbaceous plant native to Mediterranean countries, Pakistan, and India. ${ }^{1}$ The black seed has been used as natural remedy for more than 2,000 years to promote health and to treat diseases. The seed oil has been used in Arab traditional herbal medicine for the treatment of arthritis, lung diseases, and hypercholesterolemia. ${ }^{2}$ Studies have shown that the biological activity of Nigella sativa seed oil is mainly attributable to thymoquinone (TQ), a naturally occurring quinone (2-isopropyl-5-methylbenzo-1,4-quinone). TQ is the 
predominant active lipophilic component $(30 \%-48 \%)$ of Nigella sativa seed oil. ${ }^{3,4}$

TQ has a variety of pharmacological properties, such as antioxidant, anti-inflammatory, and anticancer activities. ${ }^{5}$ Numerous in vitro and in vivo studies have provided ample evidence that TQ could prevent and inhibit tumorigenesis through different molecular mechanisms, including antiproliferation, apoptosis induction, cell cycle arrest, reactive oxygen species generation, and antimetastasis. ${ }^{6}$ The antioxidant capability of TQ has been implicated in the prevention of chemical-induced carcinogenesis. ${ }^{7}$ Moreover, many studies have shown that the growth inhibitory effect of TQ is specific to cancer cells while it is minimally toxic to normal cells. ${ }^{8,9}$

Although TQ has tremendous potential as a therapeutic compound for cancer, the effectiveness and oral bioavailability are limited by its lipophilicity and poor solubility in water. ${ }^{2,10}$ Similar limitation has also occurred in cancer therapeutic drugs, such as doxorubicin, paclitaxel, and vincristine. ${ }^{11}$ The traditional practice of repeated oral administration of high doses of a drug has caused adverse side effects and less patient compliance. ${ }^{12}$ One of the alternative ways to overcome this problem is by the application of nanotechnology. ${ }^{11}$ Nanocarriers have emerged as a new technology to enhance the solubility of drugs in aqueous solution, increase their bioavailability, and enhance serum half-life, in addition to enabling tumor cell targeting and bioimaging. ${ }^{13,14}$ Some examples of nanocarriers are micelles, liposomes, micro- and nanopolymeric particles, and more recently, molecular carriers such as medicated nanofibers, ${ }^{15}$ carbon nanotubes, ${ }^{16}$ cyclodextrins, solid lipid nanoparticles (SLNs), nanostructured lipid carriers (NLCs), and lipid nanocapsules, which have significant effects on the pharmacokinetics and pharmacodynamics of bioactive compounds. ${ }^{13}$

As TQ is a hydrophobic molecule, many attempts have been made to synthesize soluble TQ analogs or encapsulate TQ in nanoformulations. Of all the nanomaterials, lipid nanoparticles offer a number of advantages such as bioacceptable and biodegradable nature, making them less toxic as compared to other nanocarriers. ${ }^{17,18}$ The NLCs are represented as an improved generation of lipid nanoparticles, which are developed from SLNs with improved characteristics. The general idea behind the system is to "mix solid lipid matrices with spatially incompatible liquid lipids, leading to imperfection in the structure of the lipid matrix", hence enhancing the drug-loading (DL) capacity. Meanwhile, NLCs still maintain the advantages of SLNs such as biocompatibility, controlled drug release, and the possibility of production on a large industrial scale. ${ }^{19}$ Previous studies have also proven that NLCs can prolong exposure to the tumor cells, enhance the permeability and retention effect, and increase the therapeutic effect. ${ }^{20,21}$

In our previous study, ${ }^{8} \mathrm{TQ}$ has been successfully encapsulated in NLC (hereinafter referred to as TQNLC), with excellent physiochemical properties such as high encapsulation efficiency (EE), high DL capacity, particle diameter less than $100 \mathrm{~nm}$, and good stability up to 2 years. In vitro studies also have proven that TQNLC exhibited antiproliferative activity toward breast cancer cell lines (MDA-MB-231 and MCF 7) and cervical cancer cell lines (HeLa and $\mathrm{SiHa}){ }^{8}$

TQNLC has large surface area-to-volume ratio, which can lead to an alteration in biological activity, and could thus be a potential anticancer agent that holds great promise for clinical translation. Moreover, in the past 2 decades, the use of nanoparticles has increased exponentially. The aim of the drug-loaded nanoparticle is to increase the therapeutic index by enhancing the drug delivery or uptake by the target cells and reduce the toxicity of free drug to nontargeted organs. The therapeutic index is the margin between the dose needed for clinical efficacy and the dose that induces adverse side effects. Hence, toxicological evaluation is needed to determine the therapeutic index of any formulation. ${ }^{22-24}$ It is vital not only to highlight the usefulness of nanoparticles but also their potential adverse effects on humans. ${ }^{25}$ In this study, TQNLC was formulated by the method described by $\mathrm{Ng}$ et al, ${ }^{8}$ and the toxicity of oral administration of TQNLC was then determined in BALB/c mice.

\section{Materials and methods Production of TQNLC}

TQNLC was produced by a high-pressure homogenization process, as described previously by $\mathrm{Ng}$ et al. ${ }^{8}$ Briefly, lipid matrices were prepared by mixing hydrogenated palm oil (Wilfarin ${ }^{\mathrm{TM}}$ hydrogenated refined palm oil; Wilmar International Limited, Neil Road, Singapore), lecithin (Phospholipon ${ }^{\circledR}$ 90G; Lipoid GmbH, Ludwigshafen, Germany), and olive oil and then melting at $70^{\circ} \mathrm{C}$. Meanwhile, aqueous matrices of sorbitol, polysorbate 80 (Tween $^{\circledR}$ 80; Sigma-Aldrich, St Louis, MO, USA), and thimerosal in deionized water $(18.2 \mathrm{M} \Omega \cdot \mathrm{cm})$ were prepared and heated at the same temperature. TQ was added and dissolved into the lipid matrices prior to dispersion of the aqueous surfactant solution into the hot lipid matrices. After mixing both matrices, the preemulsion was obtained by high-speed stirring by Ultra-Turrax ${ }^{\circledR}$ (IKA-werke, GmBH, Germany) at $13,000 \mathrm{rpm}$ for 10 minutes at $70^{\circ} \mathrm{C}$. This preemulsion was then homogenized at 1,000 bars for 20 cycles using a highspeed homogenizer EmulsiFlex ${ }^{\circledR}$-C50 (Avestin, Mannheim, 
Germany). The emulsion was allowed to recrystallize at room temperature immediately to form TQNLC. Blank NLC was prepared in a similar way, without addition of TQ into the lipid matrices.

\section{Characterization of TQNLC}

The mean diameter, polydispersity index (PDI), and zeta potential of the blank NLC and TQNLC were determined by Zetasizer Nano ZS (Malvern Instruments GmbH, Herrenberg, Germany). Each sample was diluted with deionized water (1:9) and the mean diameter, PDI, and zeta potential were measured in triplicates. The average diameter and PDI of TQNLC were analyzed at a fixed angle of $173^{\circ}$ and at $25^{\circ} \mathrm{C}$ with the Malvern software using photon correlation spectroscopy (PCS). In order to predict the colloidal stability, the electrostatic surface charge of TQNLC was determined by laser Doppler electrophoresis using the Zetasizer Nano ZS (Malvern Instruments $\mathrm{GmbH}$ ) at pH 5.8.

\section{Determination of TQNLC EE and DL capacity}

The EE of TQ by NLC was determined by an indirect method (ultrafiltration technique). ${ }^{26}$ Free TQ was separated from TQNLC by centrifugation for 10 minutes at 2,000× $g$ using an ultrafilter (Amicon ${ }^{\circledR}$ Ultra, molecular weight cutoff [MWCO]: 10,000 Da; EMD Millipore, Billerica, MA, USA). The concentration of free TQ was then determined by highperformance liquid chromatography (HPLC) analysis. EE and DL capacity were calculated using the following equations:

$$
\begin{aligned}
& \text { EE }(\%)= \\
& \frac{\text { Total amount of TQ in TQNLC }- \text { Free amount of TQ }}{\text { Total amount of TQ in TQNLC }} \times 100 \\
& \text { DL }(\%)= \\
& \frac{\text { Amount of TQ entrapped in TQNLC }}{\text { Amount of TQ entrapped in TQNLC }+ \text { Excipients }} \times 100
\end{aligned}
$$

The HPLC analysis was performed using a Waters Alliance HPLC System (Milford, MA, USA) equipped with a photodiode array detector. The stationary phase comprised a Merck HSS-T-3 C18 $(100 \times 2.1 \mathrm{~mm}, 1.8 \mathrm{~mm})$ HPLC column maintained at $30^{\circ} \mathrm{C}$. The mobile phase consisted of a mixture of methanol (70\%) and water (30\%), which was pumped at a flow rate of $1.0 \mathrm{~mL} / \mathrm{min}$. The injection volume was $10 \mu \mathrm{L}$, and analysis was performed at $255 \mathrm{~nm}$ wavelength, with a total run time of 5 minutes. Data acquisition, data handling, and instrument control were performed by Empower Software v1.0. (Milford).

\section{Experimental animals}

The Institutional Animal Care and Use Committee (IACUC), Universiti Putra Malaysia, approved this study (reference number UPM/IACUC/AUP-R026/2014) and Canadian Council on Animal Care Guide to the Care and Use of Experimental Animals were followed. Female and male BALB/c mice of 20-30 g weight and age of 6-8 weeks were used in this study. They were housed individually in cages under standard laboratory conditions, with a period of 12-hour light $/ 12$-hour darkness cycle, at $20^{\circ} \mathrm{C}-24^{\circ} \mathrm{C}$ with $40 \%-50 \%$ relative humidity. The animals were acclimatized for 1 week before the actual experiment. They were fed with a standard chow pellet (Specialty Feeds, Glen Forrest, WA, Australia) and allowed to drink water ad libitum.

\section{Acute toxicity study}

The acute toxicity study was conducted in accordance with Organisation for Economic Co-operation and Development (OECD) 420 Guideline for Testing of Chemicals. Twentyseven female BALB/c mice were randomly assigned into nine groups $(n=3)$, which were the control, two vehicle groups, and six treatment groups of TQNLC and TQ at three fixed doses $(5,50$, and $300 \mathrm{mg} / \mathrm{kg}$ of body weight). The control received tap water. The two vehicle groups received olive oil and blank NLC, respectively, of the same volume as the treated groups. For treatment groups, TQ was diluted in olive oil, while TQNLC was diluted in deionized water. All the treatments were injected via oral administration on the first day only. The mice were monitored for 14 days for any changes in the general physical conditions such as appearance, fur condition, behavior, and mortality. The body weight was measured twice a week using a table top electronic balance (AND SK-5001WP, Higashi-Ikebukuro, Toshima-ku, Tokyo, Japan).

\section{Subacute toxicity study}

The subacute toxicity study was conducted according to OECD 407 Guideline for the Testing of Chemicals. Ninety $\mathrm{BALB} / \mathrm{c}$ mice (45 females and 45 males) were randomly divided into nine groups (five males and five females): the control group, two vehicle groups, and six treatment groups of TQNLC and TQ with three escalating doses each $(1,10$, and $100 \mathrm{mg} / \mathrm{kg}$ of body weight). The control received tap water, while the two vehicle groups received olive oil and blank NLC, respectively, at the same volume as the treated groups. For treatment groups, TQ was diluted in olive oil while TQNLC was diluted in deionized water. TQ and TQNLC were orally administered by gavage for 28 days and the mice were observed for any changes in the general physical conditions, such as appearance, fur condition, behavior, 
and mortality. The body weight was measured every 3 days using a table top electronic balance (AND SK-5001WP).

\section{Blood sampling and serum preparation}

Following the observation period, the mice were fasted 2-3 hours prior to sacrifice. They were anesthetized with ketamine-xylazine $(100 \mathrm{mg} / \mathrm{kg}: 10 \mathrm{mg} / \mathrm{kg})$ by intraperitoneal injection prior to blood sampling. The blood sample $(1 \mathrm{~mL}$ ) was collected by cardiac puncture using a $26 \mathrm{G}, 1 / 2$ " needle (Terumo ${ }^{\circledR}$, Belgium, Europe) into nonheparinized and ethylene diamine tetraacetic acid-containing tubes for biochemical and hematological analyses, respectively. The blood collected in the nonheparinized tube was then centrifuged at $10,000 \times g$ for 10 minutes to obtain serum.

\section{Hematological and biochemical analyses}

Hematological parameters were analyzed using an automated hematology analyzer (Sysmex-XT-1800, Norderstedt, Germany). The parameters measured were red blood cells, hemoglobin concentration, hematocrit, mean corpuscular volume, mean corpuscular hemoglobin concentration, white blood cells, and platelet count.

Biochemical analysis was performed using a chemical analyzer (Selectra-XL, Huizen, the Netherlands). For hepatic function, the levels of serum alanine aminotransferase (ALT), aspartate aminotransferase (AST), gamma glutamyltranspeptidase, alkaline phosphatase (ALP), conjugated bilirubin, total bilirubin, total protein, and albumin were evaluated. For renal function, the levels of blood urea nitrogen and serum creatinine were determined.

\section{Tissue sampling and gross observation}

Following blood collection, the mice were euthanized by exsanguination. The major organs such as kidneys, liver, heart, lungs, and spleen were harvested, weighed, and observed grossly. For each mouse, the organ weight-tobody weight ratio (in percentage) was calculated. The organ specimens of each mouse in all groups were then fixed in $10 \%$ formalin.

\section{Histopathological analysis}

Following fixation, the tissues were cut into smaller pieces and put into cassettes. The tissues were processed using an automated tissue processor (Leica TP 1020; Leica Biosystems Nussloch GmbH, Buffalo Grove, IL, USA), whereby the machine carries out the dehydration, clearing, and impregnating process. The processed samples were then embedded in molten paraffin with appropriate molds to support the tissue during the sectioning process. The blocks were trimmed at $16 \mu \mathrm{m}$ thickness and sectioned at thickness of $0.4 \mu \mathrm{m}$ using a microtome. The thin sections of the tissue samples were placed in a water bath and fished onto glass slides. The slides were stained with hematoxylin and eosin, followed by mounting with $p$-xylene-bis-pyridinium bromide and observed under a light microscope. At least ten fields from each slide of each group were examined to evaluate the histological changes.

\section{Statistical analysis}

All values were expressed as mean \pm SEM. Comparisons between groups were performed using one-way analysis of variance (ANOVA), followed by Tukey's multiple comparison tests using SPSS software. Value of $P<0.05$ was considered significant.

\section{Results}

\section{Characteristics of TQNLC and NLC}

TQNLC and NLC appeared as yellow transparent and milky clear emulsions, respectively, at $25^{\circ} \mathrm{C}$ (Figure 1). The physiochemical properties of TQNLC and NLC are shown in Table 1. At room temperature $\left(25^{\circ} \mathrm{C}\right)$, TQNLC has a homogeneous diameter of $33.39 \pm 0.094 \mathrm{~nm}$, while NLC is $31.30 \pm 0.041 \mathrm{~nm}$ in diameter. The PDIs of both formulations were lower than 0.2 , and the zeta potentials ranged from -6.53 to $-11.62 \mathrm{mV}$.

\section{TQNLC EE and DL capacity}

Table 2 shows the HPLC analysis of free TQ detected at a wavelength of $254 \mathrm{~nm}$ and 2.3 minutes retention time. From the analysis, $98.96 \%$ of TQ was encapsulated in the NLC, and the DL was $6.85 \%$.

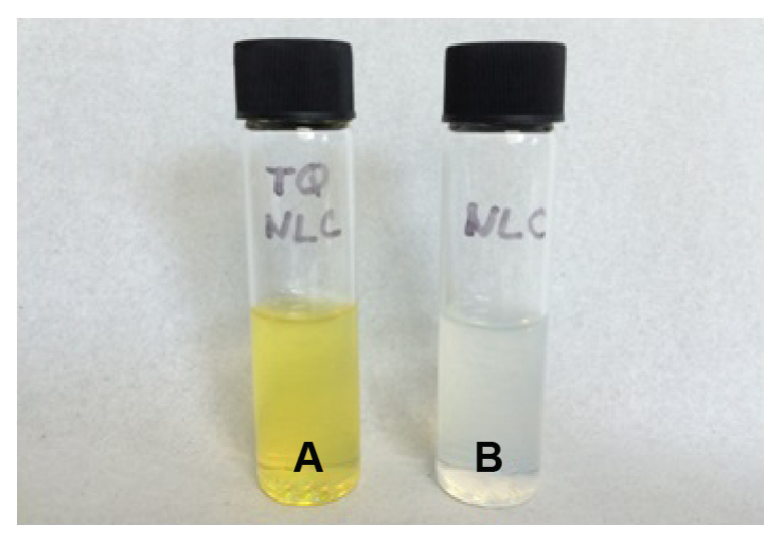

Figure I Appearance of (A) TQNLC and (B) blank NLC at $25^{\circ} \mathrm{C}$ after 24 hours. Abbreviations: NLC, nanostructured lipid carrier; TQ, thymoquinone; TQNLC, TQ-loaded NLC. 
Table I Physiochemical properties of TQNLC and NLC after 24 hours

\begin{tabular}{lllll}
\hline Formulation & Z-average $(\mathbf{d}, \mathbf{n m})$ & PDI & Zeta potential $(\mathbf{m V})$ & Temperature $\left({ }^{\circ} \mathbf{C}\right)$ \\
\hline TQNLC & $33.39 \pm 0.094$ & $0.15 \pm 0.003$ & $-10.43 \pm 0.299$ & 25 \\
NLC & $31.30 \pm 0.041$ & $0.16 \pm 0.001$ & $-11.62 \pm 0.565$ & 25 \\
\hline
\end{tabular}

Note: Values are expressed as mean \pm SEM and measured in triplicates.

Abbreviations: NLC, nanostructured lipid carrier; PDI, polydispersity index; TQ, thymoquinone; TQNLC, TQ-loaded NLC; SEM, standard error of mean.

\section{Acute toxicity study}

\section{Clinical observations and body weight change}

Table 3 summarizes the mortality rate and body weight change after treatment with TQ and TQNLC. A single oral administration of $300 \mathrm{mg} / \mathrm{kg}$ of TQ and TQNLC caused mortality to the mice. All the mice treated with $300 \mathrm{mg} / \mathrm{kg}$ of TQ died within 24 hours, while only one mouse died after 24 hours treatment with TQNLC. During the 14-day observation period, no significant weight loss $(P>0.05)$ was noted in all treatment groups as compared to the control. There was no sign of behavioral abnormality in the surviving mice.

\section{Organ weight-to-body weight ratio}

The ratios of organ weight (liver, spleen, lungs, heart, and kidneys)-to-body weight of mice in the acute toxicity study are presented in Table 4 . A single administration of TQNLC and TQ did not cause any significant changes $(P>0.05)$ in the organ weight-to-body weight ratio as compared to the control.

\section{Subacute toxicity study}

\section{Clinical observations and body weight change}

The oral administration of TQNLC and TQ for 28 days did not cause any behavioral abnormalities of the mice at any time point. No mortality was observed during the experimental period. Figure 2 shows the percentage of body weight change in the subacute toxicity study. Daily administration of TQNLC and TQ did not result in body weight loss of more than $10 \%$ in both sexes. In the female mice, the normal group showed an increase of more than $10 \%$ in the body weight.

\section{Organ weight-to-body weight ratio}

The ratios of organ weight (liver, spleen, lungs, heart, and kidneys)-to-body weight of mice in the subacute toxicity study are presented in Table 5. No significant changes
$(P>0.05)$ were noted in either male or female mice compared to the control.

\section{Hematological profile}

The effects of repeated administration of TQNLC and TQ for 28 days on serum hematological profiles are summarized in Table 6 . There were no significant changes $(P>0.05)$ in the hematological profiles of either male and female mice.

\section{Biochemical profile}

The effects of repeated administration of TQNLC and TQ on the biochemical profile (liver and kidney functions) are summarized in Table 7. Significant elevation of ALT was seen in female mice treated with olive oil as compared to the control group $(P<0.05)$.

\section{Histological changes}

No alterations were observed in the kidneys for all the treated groups in both sexes. However, pyknotic nucleus and cell degeneration were observed in the liver of mice treated with $100 \mathrm{mg} / \mathrm{kg}$ of TQ and TQNLC (Figure 3).

\section{Discussion}

This study compared the toxic effects of TQNLC and free TQ in $\mathrm{BALB} / \mathrm{c}$ mice. As described by $\mathrm{Ng}$ et al, ${ }^{8} \mathrm{TQNLC}$ was successfully prepared by the hot high-pressure homogenization technique. PCS analysis showed both NLC and TQNLC have an average diameter of less than $50 \mathrm{~nm}$ with PDI less than 0.2. According to the European Commission definition, TQNLC is considered a nanoparticle based on the particle size, which ranges from 1 to $100 \mathrm{~nm}$ with $50 \%$ or more of the particles in the size distribution. ${ }^{27}$

Particles in suspension exhibited electrostatic charges on the surface (zeta potential), which can be used to predict the

Table 2 Free TQ detected by HPLC at wavelength of $254 \mathrm{~nm}$ with retention time 2.3 minutes

\begin{tabular}{lllllll}
\hline Peak name & $\begin{array}{l}\text { Retention } \\
\text { time (minutes) }\end{array}$ & Area & \% area & Height & Amount & Unit \\
\hline Free TQ & 2.301 & 415,490 & 100 & 50,333 & 52.953 & $\mu \mathrm{g} / \mathrm{mL}^{\#}$ \\
\hline
\end{tabular}

Note: "Thymoquinone quantity in $\mu \mathrm{g} / \mathrm{mL}$.

Abbreviations: TQ, thymoquinone; HPLC, high-performance liquid chromatography. 
Table 3 Mortality rate and body weight change of female BALB/c mice treated with TQNLC and TQ in the acute toxicity study

\begin{tabular}{|c|c|c|c|c|c|}
\hline \multirow[t]{2}{*}{ Group } & \multirow[t]{2}{*}{ Mortality (\%) } & \multicolumn{4}{|c|}{ Body weight (g) } \\
\hline & & Day I & Day 5 & Day 8 & Day I3 \\
\hline Control & 0 & $20.67 \pm 0.66$ & $21.00 \pm 1.00$ & $22.00 \pm 0.58$ & $21.67 \pm 1.20$ \\
\hline Blank NLC & 0 & $19.33 \pm 0.33$ & $18.67 \pm 0.66$ & $19.33 \pm 0.33$ & $19.33 \pm 0.33$ \\
\hline Olive oil & 0 & $18.67 \pm 0.33$ & $19.00 \pm 0.58$ & $20.00 \pm 0.58$ & $19.33 \pm 0.33$ \\
\hline \multicolumn{6}{|l|}{ TQNLC } \\
\hline 5 mg/kg & 0 & $18.33 \pm 0.33$ & $18.33 \pm 0.33$ & $19.33 \pm 0.67$ & $18.67 \pm 0.67$ \\
\hline 50 mg/kg & 0 & $19.33 \pm 1.20$ & $19.33 \pm 0.88$ & $19.67 \pm 0.88$ & $20.00 \pm 1.00$ \\
\hline $300 \mathrm{mg} / \mathrm{kg}$ & 33 & $20.33 \pm 0.67$ & $20.50 \pm 0.50$ & $21.50 \pm 0.50$ & $21.50 \pm 0.50$ \\
\hline \multicolumn{6}{|l|}{ TQ } \\
\hline 5 mg/kg & 0 & $20.00 \pm 1.00$ & $19.33 \pm 1.45$ & $20.33 \pm 0.88$ & $19.00 \pm 1.00$ \\
\hline $50 \mathrm{mg} / \mathrm{kg}$ & 0 & $19.00 \pm 1.53$ & $18.67 \pm 1.33$ & $19.33 \pm 1.67$ & $19.00 \pm 1.53$ \\
\hline $300 \mathrm{mg} / \mathrm{kg}$ & 100 & $19.00 \pm 0.58$ & - & - & - \\
\hline
\end{tabular}

Notes: Values are expressed as mean \pm SEM; $n=3$. -, indicates no data obtained due to mortality.

Abbreviations: NLC, nanostructured lipid carrier; TQ, thymoquinone; TQNLC, TQ-loaded NLC; SEM, standard error of mean.

Table 4 Organ weight-to-body weight ratio of female BALB/c mice after treatment with TQ and TQNLC in the acute toxicity study

\begin{tabular}{llllll}
\hline Group & Liver & Spleen & Lungs & Heart & Kidneys \\
\hline Control & $4.67 \pm 0.39$ & $0.40 \pm 0.1 \mathrm{I}$ & $0.5 \mathrm{I} \pm 0.0 \mathrm{I}$ & $0.4 \mathrm{I} \pm 0.07$ & $\mathrm{I}$ \\
Blank NLC & $4.59 \pm 0.08$ & $0.42 \pm 0.05$ & $0.69 \pm 0.05$ & $0.45 \pm 0.12$ & $1.13 \pm 0.03$ \\
Olive oil & $4.58 \pm 0.73$ & $0.43 \pm 0.03$ & $0.59 \pm 0.01$ & $0.38 \pm 0.05$ & $1.08 \pm 0.06$ \\
TQNLC & & & & & \\
$5 \mathrm{mg} / \mathrm{kg}$ & $5.42 \pm 0.28$ & $0.49 \pm 0.07$ & $0.73 \pm 0.07$ & $0.48 \pm 0.08$ & $1.00 \pm 0.36$ \\
$50 \mathrm{mg} / \mathrm{kg}$ & $4.93 \pm 0.05$ & $0.53 \pm 0.02$ & $0.58 \pm 0.03$ & $0.43 \pm 0.04$ & $1.08 \pm 0.04$ \\
$300 \mathrm{~ms} / \mathrm{kg}$ & $4.83 \pm 0.19$ & $0.4 I \pm 0.03$ & $0.66 \pm 0.14$ & $0.46 \pm 0.08$ & $1.27 \pm 0.14$ \\
$\mathrm{TQ}$ & & & & $0.5 \mathrm{I} \pm 0.09$ & $1.23 \pm 0.08$ \\
$5 \mathrm{mg} / \mathrm{kg}$ & $4.8 \mathrm{I} \pm 0.4 \mathrm{I}$ & $0.42 \pm 0.09$ & $0.63 \pm 0.15$ & $0.46 \pm 0.03$ & $1.13 \pm 0.13$ \\
$50 \mathrm{mg} / \mathrm{kg}$ & $4.4 \mathrm{I} \pm 0.27$ & $0.42 \pm 0.05$ & $0.74 \pm 0.06$ & & \\
\hline
\end{tabular}

Note: Values are expressed as mean \pm SEM, $n=3$.

Abbreviations: NLC, nanostructured lipid carrier; TQ, thymoquinone; TQNLC, TQ-loaded NLC; SEM, standard error of measure.

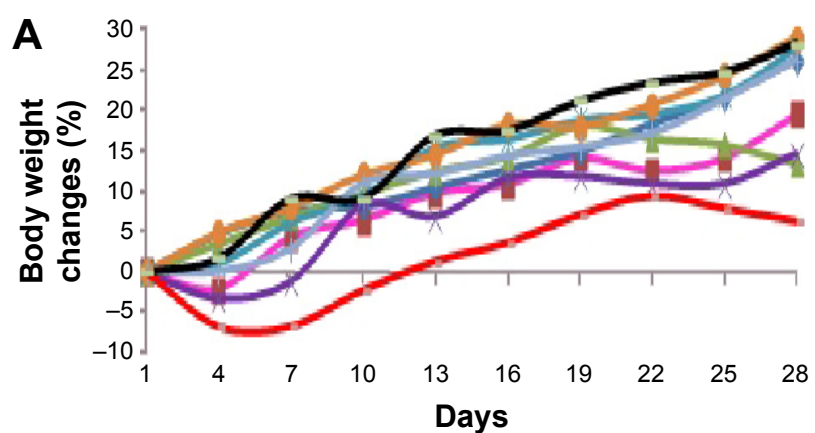

\begin{tabular}{ll|}
$\leftarrow$ NLC & - TQ: $100 \mathrm{mg} / \mathrm{kg}$ \\
$\leftarrow$ TQNLC: $100 \mathrm{mg} / \mathrm{kg}$ & - TQNLC: $10 \mathrm{mg} / \mathrm{kg}$ \\
+ TQ: $10 \mathrm{mg} / \mathrm{kg}$ & - TQ: $1 \mathrm{mg} / \mathrm{kg}$ \\
$\leftarrow$ TQNLC: $1 \mathrm{mg} / \mathrm{kg}$ & -- Normal \\
$\leftarrow$ Olive oil & \\
\hline
\end{tabular}

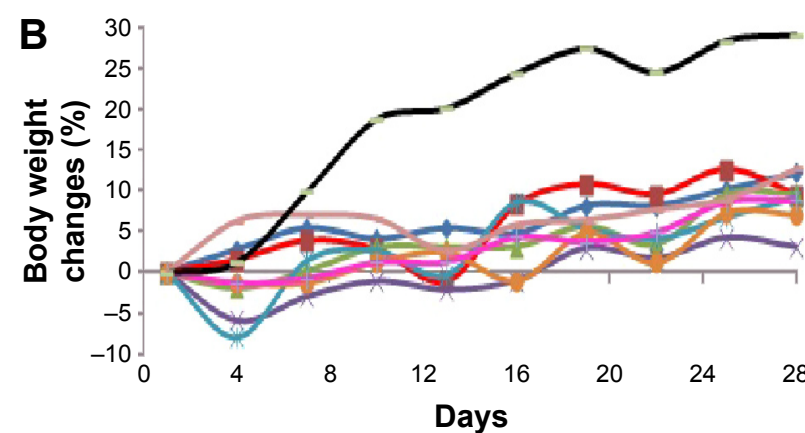

\begin{tabular}{|c|c|}
\hline$\because \mathrm{NLC}$ & TQ: $100 \mathrm{mg} / \mathrm{kg}$ \\
\hline TQNLC: $100 \mathrm{mg} / \mathrm{kg}$ & $\mp$ TQNLC: $10 \mathrm{mg} / \mathrm{kg}$ \\
\hline — TQ: $10 \mathrm{mg} / \mathrm{kg}$ & $-\mathrm{TQ}: 1 \mathrm{mg} / \mathrm{kg}$ \\
\hline$=$ TQNLC: $1 \mathrm{mg} / \mathrm{kg}$ & - - Normal \\
\hline * Olive oil & \\
\hline
\end{tabular}

Figure 2 Change in body weight of BALB/c male (A) and female (B) mice after treatment with TQ and TQNLC in the subacute toxicity study. Abbreviations: NLC, nanostructured lipid carrier; TQ, thymoquinone; TQNLC, TQ-loaded nanostructured lipid carrier. 
Table 5 Organ weight-to-body weight ratio of BALB/c mice of both sexes after treatment with TQ and TQNLC in the subacute toxicity study

\begin{tabular}{|c|c|c|c|c|c|}
\hline Group & Liver & Spleen & Lungs & Heart & Kidneys \\
\hline \multicolumn{6}{|l|}{ Male } \\
\hline Control & $3.78 \pm 0.173$ & $0.4 I \pm 0.014$ & $0.69 \pm 0.039$ & $0.45 \pm 0.026$ & $1.06 \pm 0.068$ \\
\hline NLC & $4.00 \pm 0.146$ & $0.4 I \pm 0.046$ & $0.65 \pm 0.034$ & $0.45 \pm 0.014$ & I. $30 \pm 0.058$ \\
\hline Olive oil & $3.60 \pm 0.050$ & $0.34 \pm 0.010$ & $0.56 \pm 0.017$ & $0.39 \pm 0.004$ & $1.03 \pm 0.127$ \\
\hline \multicolumn{6}{|l|}{ TQNLC } \\
\hline I mg/kg & $3.57 \pm 0.386$ & $0.40 \pm 0.013$ & $0.59 \pm 0.044$ & $0.4 I \pm 0.029$ & I. $14 \pm 0.035$ \\
\hline 10 mg/kg & $3.75 \pm 0.299$ & $0.36 \pm 0.031$ & $0.83 \pm 0.240$ & $0.45 \pm 0.044$ & $1.21 \pm 0.053$ \\
\hline $100 \mathrm{mg} / \mathrm{kg}$ & $4.08 \pm 0.126$ & $0.40 \pm 0.021$ & $0.62 \pm 0.092$ & $0.42 \pm 0.009$ & $1.19 \pm 0.043$ \\
\hline \multicolumn{6}{|l|}{$\mathrm{TQ}$} \\
\hline I mg/kg & $3.59 \pm 0.292$ & $0.33 \pm 0.021$ & $0.56 \pm 0.058$ & $0.40 \pm 0.033$ & I.I $3 \pm 0.079$ \\
\hline 10 mg/kg & $3.66 \pm 0.164$ & $0.37 \pm 0.042$ & $0.57 \pm 0.05$ I & $0.46 \pm 0.035$ & $1.16 \pm 0.058$ \\
\hline $100 \mathrm{mg} / \mathrm{kg}$ & $3.79 \pm 0.283$ & $0.36 \pm 0.014$ & $0.92 \pm 0.172$ & $0.49 \pm 0.013$ & $1.33 \pm 0.079$ \\
\hline \multicolumn{6}{|l|}{ Female } \\
\hline Control & $4.27 \pm 0.087$ & $0.58 \pm 0.028$ & $0.68 \pm 0.039$ & $0.42 \pm 0.012$ & $1.07 \pm 0.018$ \\
\hline NLC & $4.53 \pm 0.107$ & $0.54 \pm 0.042$ & $0.86 \pm 0.035$ & $0.44 \pm 0.037$ & $1.10 \pm 0.021$ \\
\hline Olive oil & $4.20 \pm 0.154$ & $0.52 \pm 0.037$ & $0.91 \pm 0.13 \mid$ & $0.45 \pm 0.013$ & $1.04 \pm 0.025$ \\
\hline \multicolumn{6}{|l|}{ TQNLC } \\
\hline $1 \mathrm{mg} / \mathrm{kg}$ & $4.27 \pm 0.195$ & $0.53 \pm 0.040$ & $0.83 \pm 0.035$ & $0.46 \pm 0.018$ & I. $13 \pm 0.033$ \\
\hline 10 mg/kg & $4.17 \pm 0.215$ & $0.50 \pm 0.020$ & $0.75 \pm 0.042$ & $0.43 \pm 0.025$ & $1.08 \pm 0.037$ \\
\hline $100 \mathrm{mg} / \mathrm{kg}$ & $4.15 \pm 0.218$ & $0.51 \pm 0.053$ & $0.72 \pm 0.043$ & $0.4 I \pm 0.017$ & $0.99 \pm 0.031$ \\
\hline \multicolumn{6}{|l|}{ TQ } \\
\hline $\mathrm{I} \mathrm{mg} / \mathrm{kg}$ & $3.97 \pm 0.184$ & $0.49 \pm 0.018$ & $0.77 \pm 0.056$ & $0.48 \pm 0.027$ & $1.07 \pm 0.043$ \\
\hline 10 mg/kg & $4.51 \pm 0.192$ & $0.50 \pm 0.032$ & $0.7 I \pm 0.037$ & $0.49 \pm 0.020$ & $1.06 \pm 0.036$ \\
\hline $100 \mathrm{mg} / \mathrm{kg}$ & $4.20 \pm 0.079$ & $0.46 \pm 0.018$ & $0.75 \pm 0.043$ & $0.45 \pm 0.016$ & $1.05 \pm 0.034$ \\
\hline
\end{tabular}

Note: Values are expressed as mean \pm SEM, $n=5$.

Abbreviations: NLC, nanostructured lipid carrier; TQ, thymoquinone; TQNLC, TQ-loaded NLC; SEM, standard error of mean.

Table 6 Hematological profile of BALB/c mice of both sexes after treatment with TQ and TQNLC in the subacute toxicity study

\begin{tabular}{|c|c|c|c|c|c|c|}
\hline Group & RBC & $\mathbf{H b}$ & PCV & MCV & WBC & Thrombocytes \\
\hline \multicolumn{7}{|l|}{ Male } \\
\hline Control & $9.11 \pm 0.38$ & $145.20 \pm 5.69$ & $0.43 \pm 0.02$ & $47.40 \pm 0.93$ & $5.92 \pm 1.35$ & $415.20 \pm 140.12$ \\
\hline NLC & $7.58 \pm 0.44$ & $118.00 \pm 5.60$ & $0.37 \pm 0.03$ & $48.00 \pm 1.14$ & $6.30 \pm 2.62$ & $277.40 \pm 202.52$ \\
\hline Olive oil & $8.15 \pm 0.71$ & $|29.60 \pm 10.4|$ & $0.38 \pm 0.03$ & $47.20 \pm 1.16$ & $4.44 \pm 1.17$ & $535.20 \pm 223.83$ \\
\hline \multicolumn{7}{|l|}{ TQNLC } \\
\hline I mg/kg & $7.28 \pm 1.16$ & $110.00 \pm 17.06$ & $0.37 \pm 0.06$ & $50.20 \pm 0.66$ & $3.76 \pm 1.05$ & $258.40 \pm 172.98$ \\
\hline 10 mg/kg & $7.86 \pm 0.68$ & $119.80 \pm 9.65$ & $0.40 \pm 0.04$ & $50.00 \pm 0.32$ & $5.98 \pm 1.38$ & $538.80 \pm 215.64$ \\
\hline 100 mg/kg & $8.24 \pm 0.28$ & $|24.20 \pm 4.5|$ & $0.42 \pm 0.02$ & $50.60 \pm 0.25$ & $5.88 \pm 1.14$ & $336.20 \pm 197.12$ \\
\hline \multicolumn{7}{|l|}{ TQ } \\
\hline I mg/kg & $8.66 \pm 0.67$ & $136.40 \pm 9.60$ & $0.4 I \pm 0.03$ & $47.80 \pm 0.20$ & $3.92 \pm 1.11$ & $308.60 \pm 171.82$ \\
\hline 10 mg/kg & $9.03 \pm 0.56$ & $142.60 \pm 9.34$ & $0.42 \pm 0.03$ & $46.60 \pm 0.68$ & $4.14 \pm 0.34$ & $440.40 \pm 169.93$ \\
\hline 100 mg/kg & $8.20 \pm 1.21$ & $134.00 \pm 17.94$ & $0.4 I \pm 0.05$ & $50.25 \pm 1.70$ & $6.40 \pm 1.69$ & $587.50 \pm 241.83$ \\
\hline \multicolumn{7}{|l|}{ Female } \\
\hline Control & $7.86 \pm 0.66$ & $125.20 \pm 9.42$ & $0.38 \pm 0.03$ & $49.20 \pm 1.07$ & $4.98 \pm 0.69$ & $441.20 \pm 200.32$ \\
\hline NLC & $8.78 \pm 0.62$ & $137.80 \pm 9.77$ & $0.4 I \pm 0.03$ & $46.40 \pm 0.87$ & $4.42 \pm 1.04$ & $357.60 \pm 162.27$ \\
\hline Olive oil & $8.7 I \pm 0.65$ & $136.60 \pm 8.29$ & $0.4 I \pm 0.03$ & $47.40 \pm 0.81$ & $5.26 \pm \mid .31$ & $637.00 \pm 229.02$ \\
\hline \multicolumn{7}{|l|}{ TQNLC } \\
\hline I mg/kg & $7.34 \pm I .4 I$ & I $14.80 \pm 20.86$ & $0.37 \pm 0.07$ & $49.80 \pm 0.49$ & $3.42 \pm 1.34$ & $403.80 \pm 221.14$ \\
\hline 10 mg/kg & $8.80 \pm 0.55$ & 1|| $.80 \pm 28.27$ & $0.44 \pm 0.03$ & $49.80 \pm 0.37$ & $4.04 \pm 1.55$ & $607.60 \pm 211.46$ \\
\hline 100 mg/kg & $8.33 \pm 0.4 \mathrm{I}$ & $|3| .00 \pm 6.94$ & $0.40 \pm 0.02$ & $47.80 \pm 0.58$ & $4.58 \pm 1.03$ & $236.60 \pm 153.80$ \\
\hline \multicolumn{7}{|l|}{$\mathrm{TQ}$} \\
\hline I mg/kg & $6.63 \pm 0.34$ & $102.50 \pm 5.49$ & $0.32 \pm 0.02$ & $48.00 \pm 1.23$ & $3.95 \pm 0.85$ & $463.00 \pm 346.45$ \\
\hline 10 mg/kg & $8.74 \pm 1.20$ & $139.00 \pm 17.90$ & $0.40 \pm 0.04$ & $46.60 \pm 1.83$ & $3.90 \pm 0.75$ & $353.60 \pm 147.66$ \\
\hline 100 mg/kg & $9.42 \pm 0.85$ & $151.00 \pm 12.83$ & $0.44 \pm 0.03$ & $46.80 \pm 1.46$ & $5.34 \pm 0.39$ & $470.00 \pm 263.97$ \\
\hline
\end{tabular}

Note: Values are expressed as mean \pm SEM, $n=5$.

Abbreviations: NLC, nanostructured lipid carrier; TQ, thymoquinone; TQNLC, TQ-loaded NLC; WBC, white blood cell; SEM, standard error of mean; Hb, hemoglobin; RBC, red blood cell; PCV, packed cell volume; MCV, mean corpuscular volume. 
Table 7 Biochemical profile of BALB/c mice of both sexes after treatment with TQ and TQNLC in the subacute toxicity study

\begin{tabular}{|c|c|c|c|c|c|c|c|c|}
\hline Group & ALT (U/L) & ALP (U/L) & AST (U/L) & $\begin{array}{l}\text { Total bilirubin } \\
\text { ( } \mu \mathrm{mol} / \mathrm{L})\end{array}$ & $\begin{array}{l}\text { Creatinine } \\
(\mu \mathrm{mol} / \mathrm{L})\end{array}$ & $\begin{array}{l}\text { Urea } \\
\text { (mmol/L) }\end{array}$ & $\begin{array}{l}\text { Total } \\
\text { protein (g/L) }\end{array}$ & $\begin{array}{l}\text { Albumin } \\
\text { (g/L) }\end{array}$ \\
\hline \multicolumn{9}{|l|}{ Male } \\
\hline Normal & $28.4 \pm 2.3$ & $290.6 \pm 8.7$ & $95.4 \pm 11.9$ & $0.72 \pm 0.36$ & $44.40 \pm 2.50$ & $11.42 \pm 1.72$ & $48.62 \pm 0.83$ & $32.62 \pm 1.02$ \\
\hline NLC & $51.1 \pm 18.9$ & $210.7 \pm 17.9$ & $201.2 \pm 141.6$ & $1.07 \pm 0.37$ & $44.17 \pm 2.40$ & $9.07 \pm 1.18$ & $45.63 \pm 3.88$ & $26.27 \pm 2.18$ \\
\hline Olive oil & $38.8 \pm 8.1$ & $280.0 \pm 19.7$ & $109.3 \pm 23.3$ & $0.75 \pm 0.26$ & $41.75 \pm 3.47$ & $8.25 \pm 0.8 \mathrm{I}$ & $44.00 \pm 0.39$ & $28.53 \pm 0.21$ \\
\hline \multicolumn{9}{|l|}{ TQNLC } \\
\hline I mg/kg & $51.1 \pm 14.0$ & $278.5 \pm 21.9$ & $143.6 \pm 22.9$ & $0.83 \pm 0.51$ & $39.50 \pm 1.48$ & $9.53 \pm 0.71$ & $45.03 \pm 1.20$ & $27.75 \pm 1.09$ \\
\hline 10 mg/kg & $20.0 \pm \mathrm{I} .7$ & $163.4 \pm 25.9$ & $104.3 \pm 20.9$ & $0.70 \pm 0.18$ & $45.60 \pm 4.01$ & $8.66 \pm 1.69$ & $44.24 \pm 1.89$ & $29.60 \pm 1.28$ \\
\hline $100 \mathrm{mg} / \mathrm{kg}$ & $39.0 \pm 5.1$ & $231.7 \pm 8.4$ & $221.4 \pm 69.1$ & $0.87 \pm 0.09$ & $39.00 \pm 0.58$ & $7.57 \pm 1.32$ & $44.00 \pm 1.06$ & $26.67 \pm 1.03$ \\
\hline \multicolumn{9}{|l|}{ TQ } \\
\hline I mg/kg & $64.1 \pm 19.7$ & $304.0 \pm 2.5$ & $196.4 \pm 55.1$ & $1.13 \pm 0.66$ & $40.67 \pm 1.67$ & $11.20 \pm 0.69$ & $46.33 \pm 0.77$ & $29.27 \pm 0.44$ \\
\hline 10 mg/kg & $36.0 \pm 15.4$ & $246.5 \pm 17.4$ & $169.3 \pm 48.6$ & $0.95 \pm 0.12$ & $54.25 \pm 9.97$ & $9.48 \pm 0.78$ & $44.95 \pm 0.92$ & $30.45 \pm 1.11$ \\
\hline $100 \mathrm{mg} / \mathrm{kg}$ & $55.9 \pm 24.4$ & $211.4 \pm 33.5$ & $229.2 \pm 91.2$ & $0.60 \pm 0.33$ & $39.80 \pm 2.33$ & $7.48 \pm 0.43$ & $49.18 \pm 3.03$ & $30.66 \pm 1.00$ \\
\hline \multicolumn{9}{|l|}{ Female } \\
\hline Normal & $53.5 \pm 8.1$ & $240.8 \pm 3.8$ & $219.2 \pm 46.4$ & $0.90 \pm 0.16$ & $40.80 \pm 1.91$ & $8.86 \pm 1.34$ & $51.70 \pm 1.83$ & $33.30 \pm 0.74$ \\
\hline NLC & $37.9 \pm 11.4$ & $173.3 \pm 4 \mid .9$ & $149.0 \pm 19.1$ & $0.63 \pm 0.35$ & $37.33 \pm 6.69$ & $7.77 \pm 1.43$ & $33.57 \pm 16.79$ & $22.20 \pm 11.10$ \\
\hline Olive oil & $205.4 \pm 80.7 * *$ & $210.3 \pm 30.0$ & $486.8 \pm 159.3$ & $0.50 \pm 0.27$ & $56.33 \pm 12.55$ & $7.77 \pm 0.99$ & $51.43 \pm 4.62$ & $28.33 \pm 1.62$ \\
\hline \multicolumn{9}{|l|}{ TQNLC } \\
\hline I mg/kg & $30.6 \pm 3.3$ & $195.7 \pm 10.1$ & $162.4 \pm 27.6$ & $1.15 \pm 0.37$ & $40.67 \pm 1.31$ & $6.55 \pm 0.61$ & $50.10 \pm 1.26$ & $30.08 \pm 0.89$ \\
\hline 10 mg/kg & $96.2 \pm 55.6$ & $177.7 \pm 4.4$ & $257.8 \pm 87.7$ & $0.60 \pm 0.07$ & $40.00 \pm 4.00$ & $7.90 \pm 1.24$ & $45.53 \pm 3.28$ & $27.73 \pm 3.37$ \\
\hline $100 \mathrm{mg} / \mathrm{kg}$ & $67.8 \pm 22.2$ & $169.8 \pm 1.7$ & $295.2 \pm 143.4$ & $0.45 \pm 0.16$ & $43.75 \pm 6.21$ & $7.70 \pm 0.93$ & $46.55 \pm 2.50$ & $29.98 \pm 2.61$ \\
\hline \multicolumn{9}{|l|}{ TQ } \\
\hline I mg/kg & $93.4 \pm 31.1$ & $213.5 \pm 8.5$ & $266.0 \pm 70.1$ & $0.80 \pm 0.10$ & $41.00 \pm 2.00$ & $7.65 \pm 0.15$ & $42.90 \pm 2.30$ & $28.20 \pm 2.60$ \\
\hline 10 mg/kg & $77.2 \pm 30.2$ & $254.8 \pm 12.1$ & $169.8 \pm 29.0$ & $0.58 \pm 0.26$ & $41.75 \pm 2.29$ & $8.50 \pm 1.31$ & $51.40 \pm 2.63$ & $33.88 \pm 0.98$ \\
\hline $100 \mathrm{mg} / \mathrm{kg}$ & $27.9 \pm 7.7$ & $245.3 \pm 15.6$ & $123.9 \pm 19.3$ & $1.05 \pm 0.13$ & $43.50 \pm 1.26$ & $9.08 \pm 1.58$ & $59.35 \pm 1.70$ & $36.32 \pm 1.37$ \\
\hline
\end{tabular}

Notes: Values are expressed as mean $\pm \mathrm{SEM}, \mathrm{n}=5$. **P $<0.01$, compared to the control.

Abbreviations: ALP, alkaline phosphatase; ALT, alanine aminotransferase; AST, aspartate aminotransferase; NLC, nanostructured lipid carrier; TQ, thymoquinone; TQNLC, TQ-loaded NLC; SEM, standard error of mean.

stability of the colloidal suspension. The high negative or positive surface charge increases the electrostatic repulsion between particles and decreases the particle aggregation. ${ }^{28}$ Zeta potentials above $30 \mathrm{mV}$ or below $-30 \mathrm{mV}$ are required for full electrostatic stabilization. However, from the zeta potential analysis, TQNLC showed a negative charge of $-11.62 \mathrm{mV}$. It has been suggested that the stability of a nanoparticle dispersion is not dominated only by electrostatic repulsion but also by the use of a steric stabilizer. ${ }^{29}$ In our previous study, ${ }^{8} \mathrm{TQNLC}$ - which is made up of a high concentration of Polysorbate 80 - was found to have stability for up to 6 months, with mean diameter less than $100 \mathrm{~nm}$. Hence, it is suggested that the stability of TQNLC is contributed by this high surfactant mixture. ${ }^{8}$

Free TQ that was not encapsulated in NLC was measured by HPLC method with C18 symmetry analytical column in a mixture of methanol and water (7:3) as the mobile phase. ${ }^{8}$ Before that, ultracentrifugation method was used to separate TQNLC and free TQ. ${ }^{28}$ From the analysis, the retention time was found to be around 2.3 minutes. High encapsulation of TQ (98.84\%) was noted. Such high EE might be due to the high lipophilicity of TQ, which caused TQ to be attracted to the lipid matrices..$^{28,30}$
TQNLC can be considered an ideal nanoformulation based on its particle size, PDI, zeta potential, and DL efficiency. These nanoparticle properties can influence the distribution of nanoparticles within the body. ${ }^{31}$ The particle size and zeta potential are the main factors affecting the nanoparticle cellular uptake, as observed for other nanoparticles such as polymeric and inorganic particles. ${ }^{28}$ The small size of the particles makes nanotechnology very useful in medicine, but it may cause adverse effects in humans. Research has shown that smaller particles are more reactive and toxic because the increase in surface area per unit mass is likely to emphasize their intrinsic properties. ${ }^{32}$

In the acute toxicity study, only female mice were used because they are generally slightly more sensitive, compared to male mice. ${ }^{33}$ The mice were orally administered with 5 , 50 , and $300 \mathrm{mg} / \mathrm{kg}$ of TQ and TQNLC on the first day only. The maximum dose level given was $300 \mathrm{mg} / \mathrm{kg}$, selected on the basis that this is the maximum volume of the formulation that can be given to the mice $(10 \mathrm{~mL} / \mathrm{kg})$. All the mice administered $300 \mathrm{mg} / \mathrm{kg}$ of TQ died within 24 hours, and no death was reported at the lower dose (50 mg/kg TQ). Thus, TQ can be classified in Category 3 in Globally Harmonized System (GHS) of Classification and Labelling 

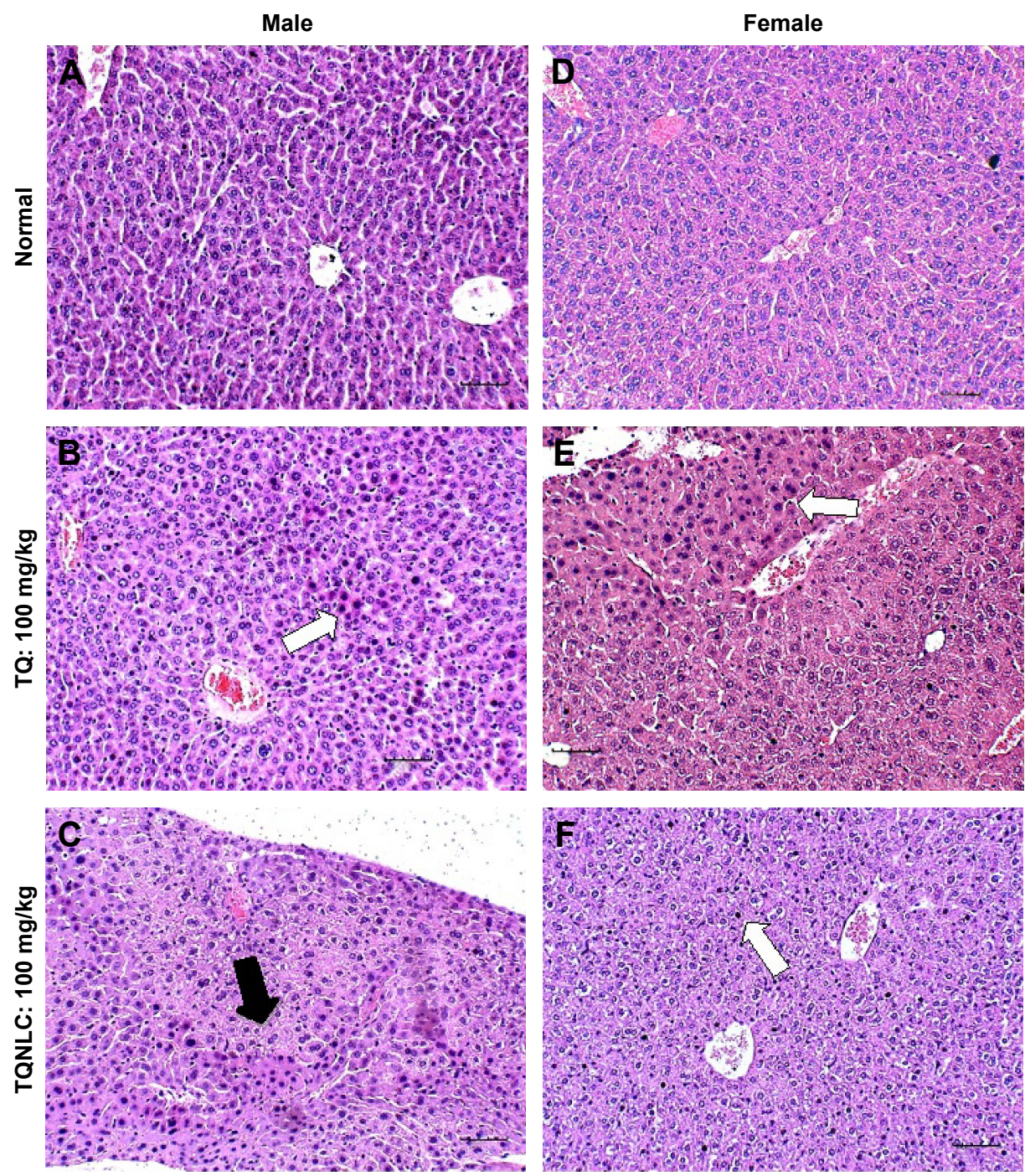

Figure 3 Liver sections of BALB/c mice after treatment with TQ and TQNLC in the subacute toxicity study.

Notes: No histological changes were observed in the mice treated with tap water (A and $\mathbf{D})$. Pyknotic nucleus (white arrows) and cell degeneration (black arrow). B and $\mathbf{E}$ show liver of mice treated with $100 \mathrm{mg} / \mathrm{kg}$ of TQ, while $\mathbf{C}$ and $\mathbf{F}$ show liver of mice treated with $100 \mathrm{mg} / \mathrm{kg}$ of TQNLC. Each scale bar represents $100 \mu \mathrm{m}$.

Abbreviations: TQ, thymoquinone; TQNLC, TQ-loaded nanostructured lipid carrier.

of Chemicals, with the anticipated oral $50 \%$ lethal dose $\left(\mathrm{LD}_{50}\right)$ falling between 50 and $300 \mathrm{mg} / \mathrm{kg}$. Delayed death was found in one mouse treated with $300 \mathrm{mg} / \mathrm{kg}$ of TQNLC after 48 hours. TQNLC was concluded to have $\mathrm{LD}_{50}$ more than $300 \mathrm{mg} / \mathrm{kg}$ but less than $2,000 \mathrm{mg} / \mathrm{kg}$, and it was thus classified as Category 4 in GHS. The determination of $\mathrm{LD}_{50}$ is usually the initial step to provide information on health hazards after short-term exposure to TQ. ${ }^{34}$ By comparing the $\mathrm{LD}_{50}$ of TQ and TQNLC, TQ loaded in NLC was found to be less toxic than pure TQ. Hence, it can be concluded that encapsulation of TQ with lipid carrier minimizes the toxicity of the compound.

In the subacute toxicity study, both male and female mice were orally administered with 1,10 , and $100 \mathrm{mg} / \mathrm{kg}$ of TQ and TQNLC. Animals of both sexes were used to evaluate the sex-based differences in response to the treatment. ${ }^{35}$ No mortality and changes in general behaviors were observed during the 28-day period. The change in body weight is used as an indicator of adverse effects of drugs and chemicals. ${ }^{36}$ Neither male or female mice lost more than $10 \%$ of the body weight and no significant changes were found in the organ weight-to-body weight ratio, suggesting no toxic effect from the treatment.

Hematological analysis has a higher predictive value for risk assessment as the hematopoietic system is one of the most sensitive targets for toxic chemicals. In this study, no significant differences were found in the hematological parameters, whereby all the values were within the normal range, suggesting that TQ and TQNLC have no effects on the blood system. 
From the biochemical analysis, the markers of kidney function (creatinine, urea, and albumin) were in the normal range for both male and female mice. The histological analysis did not reveal any significant pathological changes. Reinforcing the data, TQ and TQNLC did not induce any adverse effect on the kidneys.

Biochemical parameters such as ALT, AST, ALP, total protein, and bilirubin are the markers for liver function. In the male mice, all the liver markers were within the normal range. However, in the female mice, the group that was orally administered with olive oil had significantly elevated ALT level. ALT is the enzyme that catalyzes the reversible transfer of the $\alpha$-amino group of alanine to the $\alpha$-ketogroup of ketoglutaric acid to generate glutamate and pyruvate. ${ }^{37}$ ALT is a sensitive marker for liver injury conditions such as hepatitis, fatty liver, and cirrhosis. ${ }^{37}$ High level of ALT may suggest liver alteration by olive oil. However, this evidence of possible liver alteration should be considered together with the histopathological analysis. Liver of the female mice treated with extra-virgin olive oil did not show any histopathological changes. Research has proven that olive oil, which is a widely applied omega-9-enriched dietary lipid, exerts protective effects against chronic liver injury. ${ }^{38}$ Hence, it is suggested that the significant increase in the ALT levels might be due to the physiological response to exposure to a high concentration of fatty acid and hence not toxicologically relevant. ${ }^{39}$

The liver of both male and female mice treated with $100 \mathrm{mg} / \mathrm{kg}$ TQ and $100 \mathrm{mg} / \mathrm{kg}$ TQNLC showed areas of pyknotic nucleus and cell degeneration. Although the liver biochemical data were within the normal range, this suggests that the high dose of TQ and TQNLC may cause some toxic effects to the liver but not to the extent of altering the functions of the organ. Overall, the data also suggest that the main target for TQ and TQNLC is the liver, where the drug is metabolized and excreted via the hepatobiliary system.

In summary, oral administration of TQNLC and TQ did not cause mortality to either male or female mice but caused toxicity to the liver. It is postulated that long-term consumption of TQNLC and TQ may cause toxicity to the liver but not to the extent of altering the functions of the organ. For both treatments, the NOAEL was found to be $10 \mathrm{mg} / \mathrm{kg} / \mathrm{d}$ in mice, for both sexes. According to the formula for dose translation, the human equivalent dose of TQ and TQNLC is $0.813 \mathrm{mg} / \mathrm{kg} / \mathrm{d} .{ }^{40}$ The safety dose of TQNLC and TQ to be consumed by a healthy $60 \mathrm{~kg}$ adult would be $48 \mathrm{mg} / \mathrm{d}$.

\section{Conclusion}

Based on the acute toxicity study, encapsulation of TQ within lipid carrier minimizes the toxic effect of the compound. Long-term oral consumption TQ and TQNLC at the dose of $10 \mathrm{mg} / \mathrm{kg}$ is safe in mice and does not exert any toxic effect. The results provide safety information for TQNLC, which will further help researchers in clinical use.

\section{Acknowledgments}

This research was supported by RUGS7 (Vote no: 9359500) funded by Universiti Putra Malaysia (UPM). We would like to acknowledge Prof Dr Rasedee Abdullah and Dr How Chee Wun for their valuable contributions to this research.

\section{Disclosure}

The authors report no conflicts of interest in this work.

\section{References}

1. Gali-Muhtasib H, Roessner A, Schneider-Stock R. Thymoquinone: a promising anti-cancer drug from natural sources. Int J Biochem Cell Biol. 2006;38(8):1249-1253.

2. Khader M, Bresgen N, Eckl PM. In vitro toxicological properties of thymoquinone. Food Chem Toxicol. 2009;47(1):129-133.

3. Hajhashemi V, Ghannadi A, Jafarabadi H. Black cumin seed essential oil, as a potent analgesic and antiinflammatory drug. Phytother Res. 2004; 18(3):195-199.

4. Burits M, Bucar F. Antioxidant activity of Nigella sativa essential oil. Phytother Res. 2000;14(5):323-328.

5. Gali-Muhtasib H, Ocker M, Kuester D, et al. Thymoquinone reduces mouse colon tumor cell invasion and inhibits tumor growth in murine colon cancer models. J Cell Mol Med. 2008;12(1):330-342.

6. Ali Salim LZ, Othman R, Abdulla MA, et al. Thymoquinone inhibits murine leukemia WEHI-3 cells in vivo and in vitro. PLoS One. 2014; 9(12):e115340

7. Jrah Harzallah H, Grayaa R, Kharoubi W, Maaloul A, Hammami M, Mahjoub T. Thymoquinone, the Nigella sativa bioactive compound, prevents circulatory oxidative stress caused by 1,2-dimethylhydrazine in erythrocyte during colon postinitiation carcinogenesis. Oxid Med Cell Longev. 2012;2012:6.

8. Ng WK, Saiful Yazan L, Yap LH, Wan Nor Hafiza WA, How CW, Abdullah R. Thymoquinone-loaded nanostructured lipid carrier exhibited cytotoxicity towards breast cancer cell lines (MDA-MB-231 and MCF-7) and cervical cancer cell lines (HeLa and SiHa). Biomed Res Int. 2015;2015:10.

9. Schneider-Stock R, Fakhoury IH, Zaki AM, El-Baba CO, GaliMuhtasib HU. Thymoquinone: fifty years of success in the battle against cancer models. Drug Discov Today. 2014;19(1):18-30.

10. Pathan SA, Jain GK, Zaidi SMA, et al. Stability-indicating ultraperformance liquid chromatography method for the estimation of thymoquinone and its application in biopharmaceutical studies. Biomed Chromatogr. 2011;25(5):613-620.

11. Gabizon A, Bradbury M, Prabhakar U, Zamboni W, Libutti S, Grodzinski P. Cancer nanomedicines: closing the translational gap. Lancet. 2014;384(9961):2175-2176.

12. Yu D-G, Li X-Y, Wang X, Yang J-H, Bligh SWA, Williams GR. Nanofibers fabricated using triaxial electrospinning as zero order drug delivery systems. ACS Appl Mater Interfaces. 2015;7(33):18891-18897. 
13. Hierrezuelo J, Peláez L, Benavente J, Manuel Juan L-R, Rico R, Armengol R. Bioengineered nanomaterials. In: Tiwari A, editor. Lipid Cyclodext Nanocarriers Loadind Bioact Agents Stab Polym Support. Florida: CRC Press; 2013:226.

14. Dehghani H, Hashemi M, Entezari M, Mohsenifar A. The comparison of anticancer activity of thymoquinone and nanothymoquinone on human breast adenocarcinoma. Iran J Pharm Res. 2015;14(2): 539-546.

15. Yu D-G, Zhu L-M, Branford-White CJ, et al. Solid dispersions in the form of electrospun core-sheath nanofibers. Int J Nanomedicine. 2011;6:3271-3280.

16. Rodrigues BVM, Leite NCS, Cavalcanti B, et al. Graphene oxide/ multi-walled carbon nanotubes as nanofeatured scaffolds for the assisted deposition of nanohydroxyapatite: characterization and biological evaluation. Int J Nanomedicine. 2016;11:2569-2585.

17. Rawat M, Singh D, Saraf S, Saraf S. Lipid carriers: a versatile delivery vehicle for proteins and peptides. Yakugaku Zasshi. 2008;128(2): 269-280.

18. Kaur IP, Bhandari R, Bhandari S, Kakkar V. Potential of solid lipid nanoparticles in brain targeting. J Control Release. 2008;127(2): 97-109.

19. Lin X, Li X, Zheng L, Yu L, Zhang Q, Liu W. Preparation and characterization of monocaprate nanostructured lipid carriers. Colloids Surf A Physicochem Eng Asp. 2007;311(1-3):106-111.

20. Zhang XY, Qiao H, Ni JM, Shi YB, Qiang Y. Preparation of isoliquiritigenin-loaded nanostructured lipid carrier and the in vivo evaluation in tumor-bearing mice. Eur J Pharm Sci. 2013;49(3): 411-422.

21. Maeda H, Wu J, Sawa T, Matsumura Y, Hori K. Tumor vascular permeability and the EPR effect in macromolecular therapeutics: a review. J Control Release. 2000;65(1-2):271-284.

22. Buxton DB, Lee SC, Wickline SA, Ferrari M; National Heart, Lung, and Blood Institute Nanotechnology Working Group. Recommendations of the National Heart, Lung, and Blood Institute Nanotechnology Working Group. Circulation. 2003;108(22):2737-2742.

23. European Technology Platform on Nanomedicine. Vision Paper and Basis for a Strategic Research Agenda for Nanomedicine. Luxembourg: Official Publications of the European Commission; 2005:37.

24. Ferrari M. Cancer nanotechnology: opportunities and challenges. Nat Rev Cancer. 2005;5(3):161-171.

25. Yildirimer L, Thanh NTK, Loizidou M, Seifalian AM. Toxicology and clinical potential of nanoparticles. Nano Today. 2011;6(6):585-607.

26. Varshosaz J, Hassanzadeh F, Sadeghi H, Andalib S. Synthesis of octadecylamine-retinoic acid conjugate for enhanced cytotoxic effects of 5-FU using LDL targeted nanostructured lipid carriers. Eur J Med Chem. 2012;54:429-438.
27. European Union Executive. Commission recommendation of 18 October 2011 on the definition of nanomaterial. Off J Eur Union. 2011;L275:1.

28. Paini M, Daly SR, Aliakbarian B, et al. An efficient liposome based method for antioxidants encapsulation. Colloids Surf B Biointerfaces. 2015;136:1067-1072.

29. Thatipamula R, Palem C, Gannu R, Mudragada S, Yamsani M. Formulation and in vitro characterization of domperidone loaded solid lipid nanoparticles and nanostructured lipid carriers. Daru. 2011;19(1): 23-32.

30. Müller RH, Radtke M, Wissing SA. Solid lipid nanoparticles (SLN) and nanostructured lipid carriers (NLC) in cosmetic and dermatological preparations. Adv Drug Deliv Rev. 2002;54(suppl 1):S131-S155.

31. Montaner J, Cano-Sarabia M, Simats A, et al. Charge effect of a liposomal delivery system encapsulating simvastatin to treat experimental ischemic stroke in rats. Int J Nanomedicine. 2016;11:3035-3048.

32. El-Ansary A, Al-Daihan S. On the toxicity of therapeutically used nanoparticles: an overview. J Toxicol. 2009;2009:754810.

33. OECD. Test No. 420: Acute Oral Toxicity - Fixed Dose Procedure. OECD Publishing; 2001. Available from: https://ntp.niehs.nih.gov/ iccvam/suppdocs/feddocs/oecd/oecd_g1420.pdf. Accessed June 3, 2016.

34. Wang X, Su S, Ihsan A, et al. Acute and sub-chronic toxicity study of diaveridine in wistar rats. Regul Toxicol Pharmacol. 2015;73(1):232-240.

35. OECD. Test No. 407: Repeated Dose 28-Day Oral Toxicity Study in Rodents. OECD Publishing; 2008. Available from: http://www.oecd. org/env/test-no-407-repeated-dose-28-day-oral-toxicity-study-inrodents-9789264070684-en.htm. Accessed June 3, 2016.

36. Teo S, Stirling D, Thomas S, Hoberman A, Kiorpes A, Khetani V. A 90-day oral gavage toxicity study of d-methylphenidate and d,1methylphenidate in Sprague-Dawley rats. Toxicology. 2002;179(3): 183-196.

37. Hu X, Cheng S, Liu X, et al. Development of monoclonal antibodies and immunochromatographic lateral flow device for rapid test of alanine aminotransferase isoenzyme 1. Protein Expr Purif. 2016;119: 94-101.

38. Wang H, Sit W-H, Tipoe GL, Wan JM-F. Differential protective effects of extra virgin olive oil and corn oil in liver injury: a proteomic study. Food Chem Toxicol. 2014;74:131-138.

39. Meerts IATM, Verspeek-Rip CM, Buskens CAF, et al. Toxicological evaluation of pomegranate seed oil. Food Chem Toxicol. 2009;47(6) 1085-1092.

40. Center for Drug Evaluation and Research. Guidance for Industry Estimating the Maximum Safe Starting Dose in Initial Clinical Trials for Therapeutics in Adult Healthy Volunteers. US Dep Heal Hum Serv; 2005:1-27.
International Journal of Nanomedicine

\section{Publish your work in this journal}

The International Journal of Nanomedicine is an international, peerreviewed journal focusing on the application of nanotechnology in diagnostics, therapeutics, and drug delivery systems throughout the biomedical field. This journal is indexed on PubMed Central, MedLine, CAS, SciSearch $\AA$, Current Contents ${ }^{\circledR} /$ Clinical Medicine,

\section{Dovepress}

Journal Citation Reports/Science Edition, EMBase, Scopus and the Elsevier Bibliographic databases. The manuscript management system is completely online and includes a very quick and fair peer-review system, which is all easy to use. Visit http://www.dovepress.com/ testimonials.php to read real quotes from published authors. 